\title{
High-efficiency mission planning and distribution system of the land observation satellites
}

\author{
Qiu Hu \\ China Center for Resource Satellite Data \& Application, Beijing 100830, China
}

Email address:

tigerqiuchina@hotmail.com, tigerqiuchina@gmail.com

\section{To cite this article:}

Qiu Hu. High-Efficiency Mission Planning and Distribution System of the Land Observation Satellites. American Journal of Aerospace Engineering. Vol. 1, No. 3, 2014, pp. 16-20. doi: 10.11648/j.ajae.20140103.11

\begin{abstract}
The mission planning of earth satellite observation generally refers to the optimized use of satellites' payloads. According to relevant experiences in project construction, this paper puts forth the concept of the integrated hub-style mission planning on the basis of consumers' satisfaction and data's timeliness. This concept takes the ground processing center as the integrated service point, and data demands of difference levels of users as the driving force. It fully considers the practical problems like station resources construction lag and data transmission link frequency interference conflicts, reasonably plan the satellite payloads data reception stations resources, monitor and control resources and data processing resources, so as to implement the remote sensing data from a data request, overall planning, telecontrol, data acquisition, data processing and the unified distribution in the whole process of mission planning model. This model effectively solves the problems like the difficulty to match single satellite programming model with user demands, and the low efficiency of resource use. Besides, the paper seeks to explore relevant elements, technology and techniques which are necessary in such mission planning. The author thinks that from the vintage point of systems engineering, the commercialization of satellite observation products requires the full-cycle integrated mission planning that covers the period from the beginning of a user's order to the distribution of final data products, among which dispatching satellites' payloads is only one of important links. During the cycle, activities and constraints related to land support systems and other elements should also be taken into consideration. The comprehensive consideration including points of data acquisition, processing, archiving and distribution will make mission planning more complicated, which needs to be studied theoretically and practically. The full-cycle and integrated hub-style mission planning coordinates with national policy of civil remote sensing satellites. It is of great significance to deliver remote sensing products with high quality and high timeliness to every professional user through remote sensing data planning and major processing points, which demonstrates in two aspects. The first aspect is that it helps to achieve the win-win situation where different users get what they want and the state reduces investment in this field. The other one is that it is facile to monitor various satellite data and in return promote technology advancement of satellite processing and construction, which eventually stimulates the development and progress of aerospace remote sensing technology.
\end{abstract}

Keywords: Mission Planning, Land Observation Satellite, Hub-Style, Full-Cycle, Frequency Interference, Overall Planning

\section{Introduction}

Because of its superiority in data acquisition, the observation satellite is playing a crucial role in all aspects of social development. With the maturity of satellite manufacture technology and constant development of aerospace equipment, China is applying Earth observation satellites in a more commercialized way. Thus, with the help of mission planning, how to maximize resources utility and users' satisfaction through overall management of producing of land observation products? At present, both at home and abroad satellite mission planning technology has received mass attention and has achieved admirable success in this field though, the concept of mission planning has been confined to the optimized operation of satellite payloads[1-3]. This paper from the aspect of systematic engineering, holds that the commercialization of satellite observation products requires the full-cycle integrated mission planning from users' requests to the final data distributed to users, of which the scheduling of satellite payloads is just one of important links. Besides, we should consider activities and constraints associated with the ground support systems and other factors including the deadline of distributing products to customers, the meteorology of observing regions, the constraints on the 
capability of satellite payloads, the limit of ground data transmission capability, the task allocation and the effective use of the distributed ground receiving systems, the flexibility of handling changes and unexpected events, and the frequency interference between different ground links. The whole management of data collection, processing, archiving and distribution make mission planning even more complicated, which needs to be explored further theoretically and practically, so that a number of elements should be considered, and a set of advanced techniques should be referred to.

\section{The Full-Cycle Mission Planning}

\subsection{Fulfillment of Full-Cycle Satellite Application Mission}

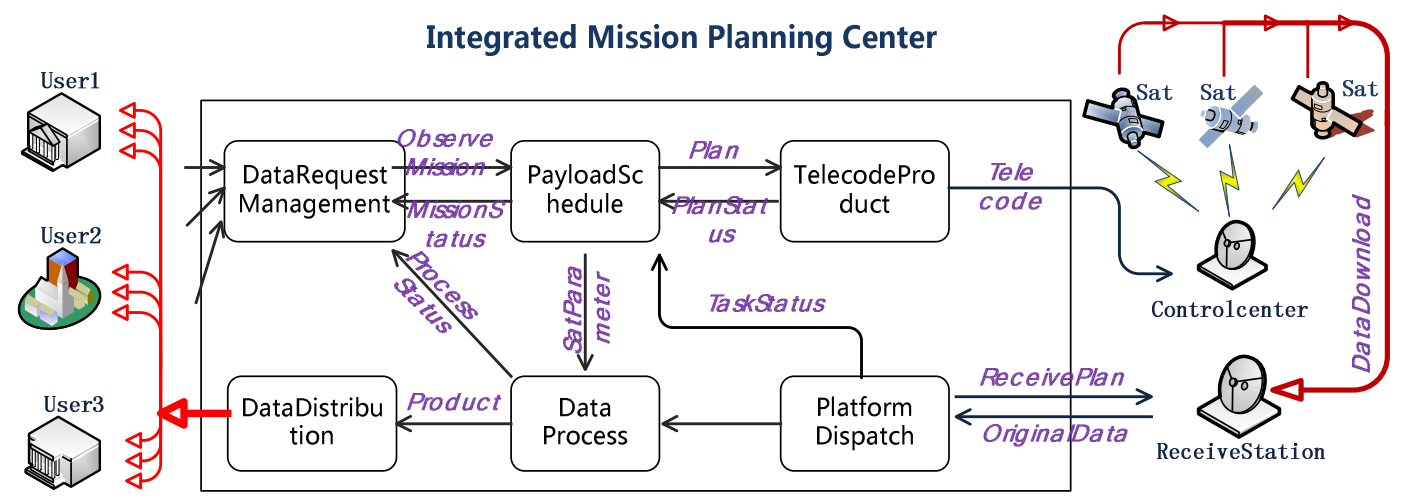

Figure 1. Full-cycle satellite application mission system model

A fulfilled satellite application mission is a circulating from the submission of users' requests to the distribution of data products. As shown in the chart 1 , the process is as follows.

(1) Provide users with satellite observation planning through the Internet;

(2) Receive users' observation request through the Internet and the like;

(3) Preliminarily examine the feasibility of users' requests;

(4) Put users' requests into the payloads planning optimization module to avoid the conflict in resource use;

(5) Transform the planning results into remote commands towards satellite payloads;

(6) Check availability of other resources related to data acquisition, such as TDRSS satellites;

(7) The planning of ground support activities, including moderation made by the availability of resources and priority of different missions, to the scheduling of ground receiving activities, the data processing, and the scheduling of data distribution;

(8) Acquire, receive and process data;

(9) Send situations feedback to users;

(10)Archive and distribute data products;

(11)Users receive ordered products.

Among the steps above, (4) and (7), where resources use conflicts are most likely to happen thus mission planning is needed, are of the greatest importance. Traditional satellite mission planning always focuses on step (4). The result of planning corresponds to the sequence of satellites' observation activities, which is described in the nest section.

\subsection{The Traditional Concept of Satellite Mission Planning}

Traditional satellite planning, usually interpreted as optimal use of satellite payloads, aims to schedule the space-borne sensor resources and specific time for each observation activity on the basis of the constraints on satellite payloads and requirements for observing missions, in order to maximize users' satisfaction. Relevant constraints should be considered by traditional planning include:

(1) The constraint of satellite system's capability Elements, including energy and power, the number and the type of sensors, capacity of data storage, and the time to change the angle of side-sway, would hinder the performance of observations.

(2) The constraint of the time window When satellites observe objects or transmit initial data down to the land reception station, all tasks should be finished within limited time window. Some objects could not be observed.

(3) The constraint of weather conditions For example, visible light shooting has strict restraints on cloud thickness.

(4) Special requirements for certain missions Special constraints like periodical or paired observations are needed in some missions.

Traditional mission planning could only decide when to acquire initial data, while it could not determine whether the acquired data could be transferred to land stations without delay, or when the data could be processed and distributed to users successfully. As land observation satellites undergo the transition from experimental use to more practical and commercial use, users have more strict requirements on the quality of data products and the efficiency of collecting those products, for example, at some users' request, the time span, from high resolution imaging of the targets to the submission of data products should be less than a few hours. Typically the stricter the requirements are, the more profits are achieved by observing missions. Finally, application systems of land 
observation satellites shall provide consumers with continuous information service instead of occasional data products. However, traditional satellite mission planning cannot fulfill this kind of task. To solve the problem, this paper introduces the concept of full-cycle mission planning.

\subsection{The Intension of Full-Cycle Mission Planning}

From the perspective of systematic engineering, full-cycle mission planning considers the whole process of satellite application task, including data acquisition, downlink, processing and distribution, as integral. By scheduling all activities in a integrated way, the planning endeavors to maximize users' satisfaction and satellites' use efficiency. Highlighting the quality of service, the full-cycle mission planning fully considers users' requirements on products, which contributes to commercialization of satellite data products and users' satisfaction.

Compared to traditional mission planning, some other elements should be added in the full-cycle mission planning:

1. The change of distribution system While the ground support system is previously designed, the distribution system can be changed all the time. In that way, the service for consumers and the planning should be more flexible.

2. The mechanism of ground support system The ground support system usually comprises a number of land stations with similar functions and processing centers. Its planning also requires coordination and distributed handling, and also involves specific constraints.

3. The connection to the external data communication network Data distribution requires a communication network covering large areas, which is also a part of the full-cycle. Therefore, special requirements and constraints towards the connection between ground support system and communication network are involved.

4. Automatic operation and operating cost saving The trend of modern technology development is to save operating cost by promoting automation of operations, while weighing automation against technical risks. To achieve the goal of full-cycle mission planning, we can make full use of the progressive technology satellite payloads, for example, the solid-state storage devices with large capacity can increase the flexibility of data recording and downlinking, efficient power usage can prolong the service cycle of certain equipments, TDSSR satellites can improve amount of sent-back data, and technology of mass data downlinking at high speed improves the ability of missions accomplishment. At the same time, the scope of traditional mission planning shall be extended. Planning technology needed to be innovated, and only by combining hard technology and soft technology can maximize the profits. The following section will mainly describe the full-cycle mission planning from the perspective of soft technology.

\section{Elements to Realize Full-Cycle Mission Planning}

\subsection{User Interface}

User interface is the direct channel of communication between users and satellite application systems. Fine user interface can enhance users' satisfaction of the service. Firstly, user interface should provide users with convenient consulting product database. When current database can meet users' requests, the interface then should permit them to download the data. When existing products fail to meet users' needs, the interface could supply standard formats and effective guidance for user's inputting new observation requests, so that the full-cycle mission planning can analyze the request and extracting mission-related information. Secondly, in view of different locations of the data users, the interface should apply distributed features, that is, the service meant for users' terminal should have a unified view that does not change with locations. What is more, to satisfy the need of near-real-time products, more customer service centers can be established in accordance with the layout of data storage, processing centers and ground stations. Data products are distributed by external communication network. Users can constantly submit orders or receive data products in a recycled way by the same network [4].

In addition, when the system is handling their requests, the interface should allow users to keep track of the progress. In particular, the cancellation of order and false information must be fed back to users in time. Thus, the interface should encompass monitoring mechanism of contingency in data distribution as well as the identification of the infeasible requests.

\subsection{The Identification of Missions and Constraints}

Identifying all detailed requirement and related constraints is the premise of full-cycle mission planning. Users' observation orders may vary, so different satellite resources and different constraints are involve. For example, some users demands visible light imaging, indicating that the image should be taken by visible light imaging satellites under favorable sunlight conditions; some users may require all-weather observation, where SAR satellites are used. Some global scale missions require regular target observation and data downlink in every circle of satellites' flight to obtain continuous data products, where satellites with low resolution can manage. But in regional missions, it is satellites with high resolution that implement observation and downlink the data to ground station or TDRSS satellites. Requirements for the service are always changing, among which the near-real -time trait becomes the main constraint in the planning of data acquisition, processing and distribution.

\subsection{The Planning of Satellite Payloads}

It is difficult to figure out a unified model and an efficient scheduling algorithm of multi-type satellites and their 
payloads based on constraints in satellite payloads planning. To give prominence of the advantages of full-cycle mission planning, it is necessary to schedule the various satellites in a unified and coordinated way. As for the different operating constraints of various satellites, such as visible light imaging satellites and SAR imaging satellites, they have to be described in a unified form in the integrated planning schedule model.

There are other aspects in scheduling algorithm need considering. If we simply follow the principle of First Come First Served, we may obtain a feasible solution, but it may not maximize the profits or users' satisfaction. The planning of satellite payloads is a combinatorial optimization problem with NP trait, which suggests that it is difficult to attend both the time constraint and the optimal solution of the algorithm. Therefore, the scheduling algorithm ought to strike a balance between solution performance and solution time to satisfy users' requirements in terms of the priority and time span of different observing missions.

\subsection{The Planning of Ground Support Systems}

According to its structure, mission planning of ground support system can be divided into two stages--data processing and control center stage and distributed ground stations stage. Stage two after preliminarily processing the data downlinked from satellites, sends the data to the former stage, and then the former processes data again and delivers final products to users. The planning cycle of stage one is rather longer (1 2 days), while the cycle of stage two depends on the circle of the satellite. The planning of two stages have to solve all conflicts in resource utilization related to data processing and distribution, as well as allow addition, revision and deletion of specific data products. A good number of departments and links are involved in the problem of ground support system planning, of which the scheduling of data processing is quite crucial. Because the same processing chains need to tackle offline missions, such as the additional processing of products and the disposition of overstocked missions, at the interval of finishing near-real-time data processing missions.

Furthermore, other elements should be considered in the scheduling of data processing, for instance, the test of capacity and feasibility of data processing chains, the allocation of the operating loads to different processors, and the automation of mission planning. The large number of products also contributes to the complexity of ground support planning, let alone the combination of processing and distribution of the products, which resulted from the different types of data processing activities (including near-real-time processing, offline processing, and overstocked mission handling), the site of data process(generally there are several data processing centers or stations.), the type of products(including regular products of global missions or occasional products of regional mission), the media for data distribution(such as solid-state recorders, wireless broadcast communication links, and multicast links), and the targets of data distribution(users or processing and control centers). The aspects of ground support systems mentioned above add to the difficulty of full-cycle satellite land observation mission planning.

\subsection{Data Distribution}

Data distribution is the last link of full-cycle mission plan. Users' satisfaction largely depends on whether they could receive ordered products within given time. Since the data processing center and the user who submits requests are not always at the same place, both near-real-time data products and regular mission products are delivered through particular distributed data communication networks. What is more, the mass amount of satellite observing data could easily lead to network congestion or interrupt, thus may delay users obtaining the products and decrease their satisfaction. Hence compression technique of massive information is significant to lessen the total amount without much information loss. After compression, data products are saved in remote servers and system will provide operating port to remote users.

In light of the limited bandwidth and transmission rate of current network system, proper transmission strategy and agreement should be reached to accelerate mass information transmission. Generally, data of smaller amount can be transmitted through broadcast communication links with relative low speeds. As for the massive data, multicast data links have more advantages. Also, if massive data is required to be transmitted within a rather short period, other high-speedy transmission channels can be utilized.

\subsection{Relevant Technology and Techniques}

A practical full-cycle mission planning software has to be flexible enough to facilitate the addition, moderation, and deletion of rules and constraints. Meanwhile, the software needs to maintain interaction with planners, while the final decision to solve the conflicts comes to planners. The expert system requires identification and authentication mechanism, so it is not applicable to planning and scheduling. Traditional operational researches methods like mathematic programming could not manage such a complicated problem either, whether in modeling or seeking the proper solution.

Given the development of planning and scheduling software outside of China, the algorithm of constraint-based scheduling is the most feasible. Constraint-based scheduling algorithm transforms scheduling problem into Constraint Satisfaction Problem(CSP) in artificial intelligence and applies relative Constraint Programming $(\mathrm{CP})$ to modeling and scheduling. CSP problem can be defined by the triple combination of a variable set, a variable value set and a constraint set of variable values, and its solution should be a value combination of variables that meets all constraints. If required, the given target should be optimized. CP inaugurates a highly effective way to solve CSP--its programming language can describe a host of CSP models, and its constraints processing algorithms and predefined search algorithm library guarantee the efficiency of problem solving. For example, American GREAS satellite mission planning system employs CP tools, ILOG Solver and ILOG Scheduler, 
developed by French company OLOG, providing moulds of all missions, resources and constraints. Here, missions refer to the activities and operations to be conducted, resources are human, satellites, sensors and communication bands used in implementation, and constraints are those defining the time of mission implement, relationship between missions, and the capacity and availability of resources. Although GREAS system does not include the planning for ground support system, its solving approach is a splendid reference to full-cycle mission planning. Another point to be stressed--CP technique is different from mathematic programming in that the models do not necessarily correspond to the solving algorithms. In other words, the same model may have a variety of methods to figure out the solution, including accurate search like branch and bound algorithm, and also inaccurate heuristic search such as taboo search, and genetic algorithm. Accordingly we can make full use of $\mathrm{CP}$ approach to build flexible models in solving complex planning and scheduling problem. Meanwhile, we can design by ourselves or borrow other inaccurate search algorithms with outstanding time behavior to get satisfactory mission planning based on the exact description of problem traits.

\subsection{Prevention of Frequency Interference}

At present, most data transmission systems of remote sensing satellites use the $\mathrm{X}$ band $(8025-8400 \mathrm{MHz})$ for data downlink, they march in sun-synchronous polar orbit, their adopt the local time of descending node is 10:30, and the data is received by the same ground receiving station network. These similar parameters make frequency interference more likely to occur between different data link channel.

In the past years, one operator corresponds to one specific satellite, however, this mode could not solve the problem of frequency interference effectively. Nowadays, the "hub-style" satellite mission planning can formulate plans in advance, and then settle the problem with the optimal solution.

In accordance with the interference protection threshold of International Telecommunication Union(ITU), the calculation and analysis of interference margin of the link can make suggestions to the judgment and prevention of data link frequency interference.

\section{Conclusion}

That the application of observing satellites converts from experimental use to practical use requires the full-cycle mission planning of satellite systems and ground support systems as integral. Satellite is just one of the links in service to users and the feature of current missions embodies in the stricter requirement on the efficiency and quality of data products. New problem about management of the relevant activities around ground support system emerges as well. Since it is a long cycling chain from requests submission to products delivery, the monitoring and optimization of ground data processing and resource allocation become necessary.
Only by viewing the cycle chain of production as a whole can we satisfy users' different requirements of desirable products. When practicing full-cycle mission planning, constraint-based scheduling is a favorable method, while more detailed techniques shall be further studied.

In the last a few decades, remote sensing satellites of China have witnessed significant changes, from single number to more than a dozen, from universal usage to specialized division. The full-cycle hub-style mission planning corresponds to national policy of civil remote sensing satellites, that is, unified planning and centralized handling. It is of great significance to timely deliver products of high quality to every professional user through remote sensing data planning and major processing points--first, this mode technically resolves the planning and optimization problem of numerous satellites; second, it helps to achieve the win-win situation where different users get what they want and the cost is reduced; third, it keeps supervising and monitoring various satellite data, and in turn propels the development of satellite processing and manufacturing techniques, and systematically stimulates the development and progress of aerospace remote sensing technology.

\section{References}

[1] Payload Design Description, RapidEye internal document, RE-DD-DJO-2000,Nov.2007

[2] E.Stoll,R.Schulze,B.D'Souza, M.Oxfort,'The impact of collision avoidance maneuvers on satellite constellation management", in proc. Of European Space Surveillance Conference, Madrid, Spain,2011

[3] Nicola Bianchessi,Jean-Francois Cordeau, Jacques Desrosiers, Gilbert Laporte, Vincent Raymond. A Heuristic for the Multi-Satellite, Multi-Orbit and Multi-User Management of Earth Observation Satellites. European Journal of Operational Research.vol.177,pp.750-762,2005

[4] Xu Xue-ren,Gong Peng,Study on Optimization of Satellite.Journal of Remoter Sensing,vol.56,pp:962-968,2007

[5] H. Muraoka, R. .Cohen, T. Ohno, and N. Doi. Aster observation scheduling algorithm. In Proceedings of the International Symposium Space Mission Operations and Ground Data Systems.1998

[6] J. Pemberton. Towards scheduling over-constrained remote sensing satellites. In Proceedings of the $2^{\text {nd }}$ International Workshop on Planning and Scheduling for Space,2000.

[7] D. Smith, J.Frank, and A, Jonsson. Bridging the gap between planning and scheduling. Knowledge Engineering Review,15(1),2000.

[8] B-S Lee, J-S.Lee,B-S.Lee,and J-W.Eun, "Mission Analysis and Planning System for Korea Multipurpose Satellite-I",ETRI Journal, Vol.21,No,3,1999,pp.29-40.

[9] Zitzler E.Evolutionary algorithms for multiobjective optimization: methods and applications [D]. Zurich: Swiss Federal Institute of Technology,2002 